\title{
Graphene Promotes Axon Elongation through Local Stall of Nerve Growth Factor Signaling Endosomes
}

\author{
Domenica Convertino,* Filippo Fabbri, Neeraj Mishra, Marco Mainardi, Valentina Cappello, \\ Giovanna Testa, Simona Capsoni, Lorenzo Albertazzi, Stefano Luin, Laura Marchetti,*, \\ and Camilla Coletti*, II
}

Cite This: Nano Lett. 2020, 20, 3633-3641

Read Online

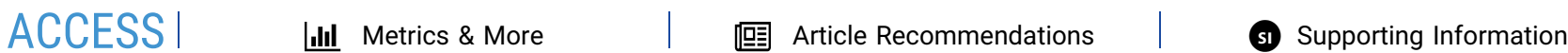

ABSTRACT: Several works reported increased differentiation of neuronal cells grown on graphene; however, the molecular mechanism driving axon elongation on this material has remained elusive. Here, we study the axonal transport of nerve growth factor (NGF), the neurotrophin supporting development of peripheral neurons, as a key player in the time course of axonal elongation of dorsal root ganglion neurons on graphene. We find that graphene drastically reduces the number of retrogradely transported NGF

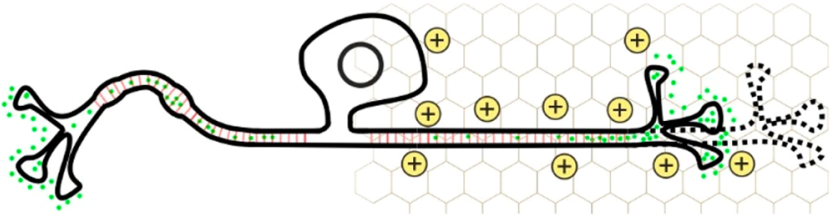

· FluoNGF $\oplus$ Holes created by electronegative cell membrane vesicles in favor of a stalled population in the first 2 days of culture, in which the boost of axon elongation is observed. This correlates with a mutual charge redistribution, observed via Raman spectroscopy and electrophysiological recordings. Furthermore, ultrastructural analysis indicates a reduced microtubule distance and an elongated axonal topology. Thus, both electrophysiological and structural effects can account for graphene action on neuron development. Unraveling the molecular players underneath this interplay may open new avenues for axon regeneration applications.

KEYWORDS: Graphene, peripheral dorsal root ganglion neuron, axon elongation, material-neuron interface, nerve growth factor retrograde transport, membrane-associated periodic skeleton

I

$\mathrm{n}$ the past years, a number of studies have investigated graphene potential as a conductive neural interface, able to enhance adhesion, proliferation, and differentiation of various cell types, including neural cells. ${ }^{1-4}$ In the last case, an interesting increased neurite sprouting and outgrowth induced by graphene were reported for hippocampal neurons, ${ }^{1}$ differentiated SH-SY5Y neuroblastoma cell line, ${ }^{5}$ adrenal phaeochromocytoma (PC12) cell line, ${ }^{4,6,7}$ dorsal root ganglion (DRG) neurons, ${ }^{4}$ and neural stem cells. ${ }^{8}$ Such a feature makes graphene appealing for application in peripheral nerve regeneration, where an appropriate scaffold may accelerate neurite outgrowth. ${ }^{9,10}$ However, to date, few studies have examined the interaction of graphene with peripheral neurons. Thus, the nanoscale mechanisms by which graphene would promote axon regeneration remain unclear.

To understand the molecular mechanism of axon outgrowth on graphene, some groups investigated GAP-43, a recognized marker of developing and regenerating axons. Increased GAP43 levels were reported in hippocampal neurons grown on graphene. ${ }^{1}$ Similarly, increased GAP-43 together with synaptophysin levels were also reported in PC12 cells grown on aligned silk-graphene hybrid hydrogels, ${ }^{6}$ suggesting that the mechanisms driving neuritogenesis may be shared for central and peripheral neurons grown on graphene. However, the underlying mechanisms for the increased GAP-43 expression on graphene, together with structure and dynamics of neuron development, have not yet been investigated. A recent study has reported an increased cell firing for hippocampal neurons grown on graphene, probably due to altered membrane ion currents at the material interface. ${ }^{11}$ Indeed, several reports described a positive effect of electrical stimulation on axonal outgrowth and branching. ${ }^{3,12-14}$ However, such an effect may not necessarily apply to peripheral neurons on graphene, as it was already reported that DRG neurons respond differently from hippocampal neurons on nanofabricated biomaterial scaffolds. ${ }^{15,16}$ Importantly, all of the above-cited studies were carried out on cultures grown from 1 to 3 weeks onto the substrate. While this strategy is useful to understand the longterm effects of the material on neuron physiology, it fails to investigate how the early developmental stage is influenced by the neuron-material interface. This appears to be an important issue to address, because the positive effect of graphene on axonal sprouting and outgrowth was found to be maximal during the first 2 days of culture and then to decrease

Received: February 10, 2020

Revised: March 24, 2020

Published: March 25, 2020 
a

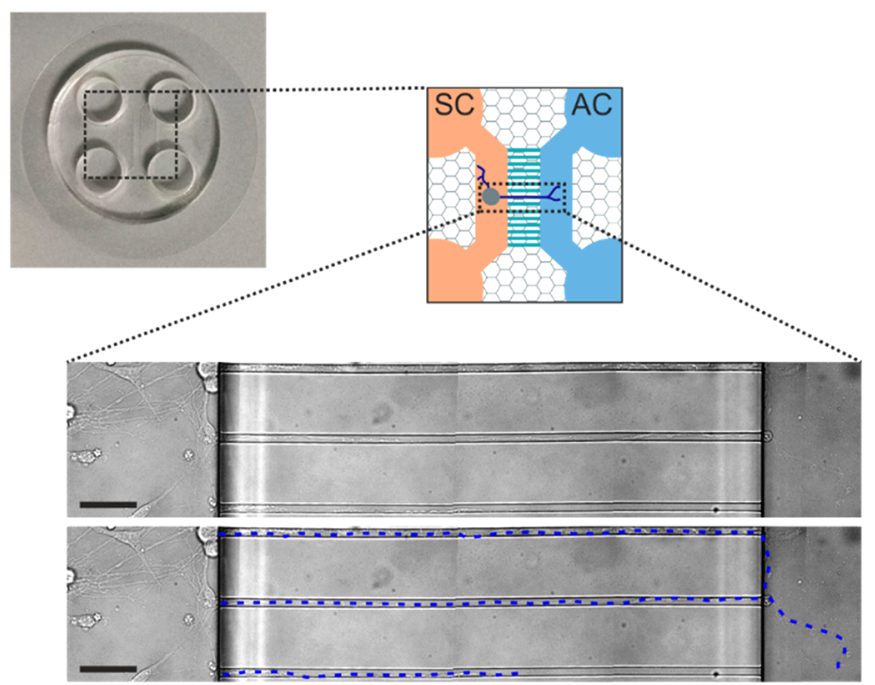

b

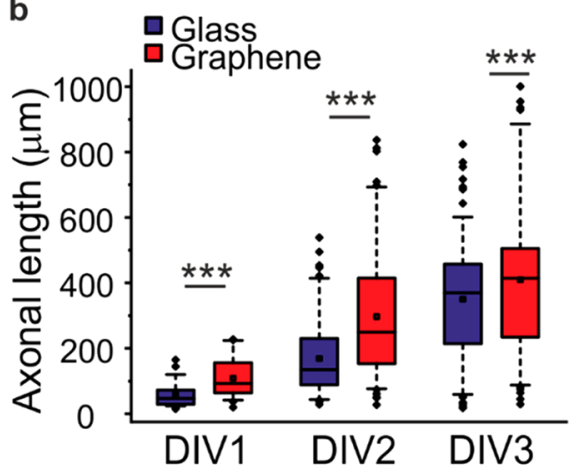

Figure 1. Increased axonal elongation in DRG neurons grown on graphene. (a) Schematic representation of DRG axonal length quantified on graphene inside a microfluidic chamber. Top left: Optical image of an assembled device on graphene. Top right: Cartoon showing the microfluidic chamber with the soma compartment (SC) and axon compartment (AC) connected by microchannels. Bottom: Bright-field images with axons crossing and filling the microchannels. The same area is reported below with the axons highlighted by dashed blue lines. Scale bars: $50 \mu$ m. (b) Quantification of the axonal length at different DIV on glass and graphene in a microfluidic device. A significant difference was found between graphene and control according to two-way ANOVA test $\left(P_{\text {substrate }}<0.001\right.$, two-way ANOVA, with Holm-Sidak posthoc test, $\left.* * * p<0.001\right)$. The number of measured axons was: DIV1, glass $n=42$, graphene $n=50$; DIV2, glass $n=109$, graphene $n=101$; DIV3, glass $n=147$, graphene $n=$ 119. In box plots: Box between 25th and 75th percentile; horizontal line: median; whiskers: 5th and 95th percentiles; square: mean; circles: outliers.

up to a steady-state level in which axons are slightly longer with respect to control cultures. ${ }^{1}$

In this work, we demonstrate that graphene significantly stimulates axonal outgrowth during the first 3 days in culture of DRG primary neurons. Hence, we investigate the effect of graphene on the fast axonal transport properties during the early developmental phase. In detail, the role of nerve growth factor (NGF), a key neurotrophin involved in neurite elongation and survival during DRG development, ${ }^{17,18}$ is investigated by single-molecule fluorescence microscopy. Remarkably, we find that axonal elongation on graphene correlates with a significant reduction of NGF vesicles retrogradely transported to the soma, in favor of a stalled pool retained locally in developing axons. Patch-clamp recordings and ultrastructural analyses concur to show that profound rearrangements occur in axons developing on graphene, which may account for the local accumulation of NGF resulting in the increased axon elongation. Our results provide a broad structural and functional understanding of the impact of graphene on DRG neurons, key information toward the development of graphene-based devices for neural regeneration applications.

\section{RESULTS AND DISCUSSION}

Graphene Promotes Axon Elongation in Developing DRG Neurons. The effect of graphene on axon outgrowth in primary peripheral neurons was investigated using DRG neurons dissected from postnatal day (P) 4 mice, ${ }^{19}$ which were cultured on PDL/laminin-coated substrates ${ }^{4}$ in the presence of NGF. We used graphene grown via chemical vapor deposition $(\mathrm{CVD})^{20}$ (for more details, see SI). In order to precisely determine axonal length, we used a compartmentalized microfluidic chamber placed on top of a graphenecoated glass coverslip (Figure 1a).
This approach allows to selectively apply treatments to axons and mimics well the physiological NGF stimulation, where the trophic factor is produced by target innervated tissues and is endocyted at the distal axon tips, far from the cell soma. $^{21,22}$

Figure $1 \mathrm{~b}$ reports the axon length distribution measured at three time points. We found gradually increasing neurite lengths both on graphene and glass, with significantly higher values on graphene. Remarkably, in the first 2 days of culture, the axons were longer on graphene by $79 \%$ and $73 \%$, respectively (Table 1 ). At day 3 , reduced although significant

Table 1. Axonal length on microfluidic chamber at different DIV $^{a}$

$\begin{array}{lcrrc}\text { substrate } & \text { DIV } & \begin{array}{c}\text { axonal } \\ \text { length } \\ (\mu \mathrm{m})\end{array} & \text { s.e.m. } & \begin{array}{c}\text { percentage increase on graphene } \\ \text { with respect to the control }\end{array} \\ \text { graphene } & \text { DIV1 } & 114.6 & 8.0 & 79.3 \% \\ \text { glass } & & 63.9 & 5.7 & 73.6 \% \\ \text { graphene } & \text { DIV2 } & 305.2 & 19.2 & \\ \text { glass } & & 175.8 & 10.7 & 16.6 \% \\ \text { graphene } & \text { DIV3 } & 419.6 & 22.0 & \\ \text { glass } & & 359.7 & 14.9 & \end{array}$

${ }^{a}$ Average \pm standard error of the mean (s.e.m.) of length values plotted in Figure $1 \mathrm{~b}$, and percentage of length increase on graphene with respect to glass at different DIVs.

differences were found, with the axons on graphene $17 \%$ longer than on glass. After day 3, the axons crossing the microchannels started to form complex networks that impeded to further quantify their length with accuracy.

It should be noted that the same axonal length analysis at an early developmental stage was also performed on standard and graphene coated coverslips, yielding results that confirm preferential neurites outgrowth on graphene (Figure S1a,b). 


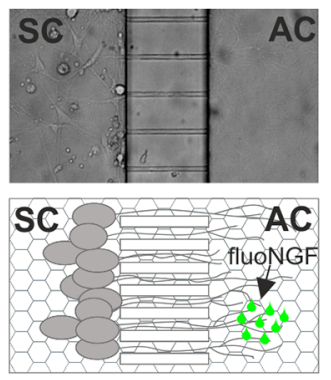

b

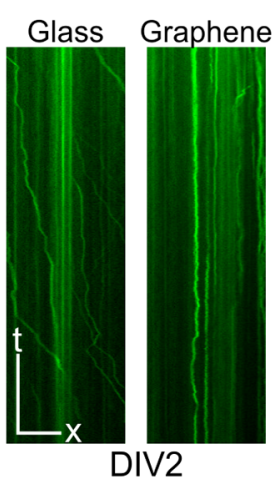

d

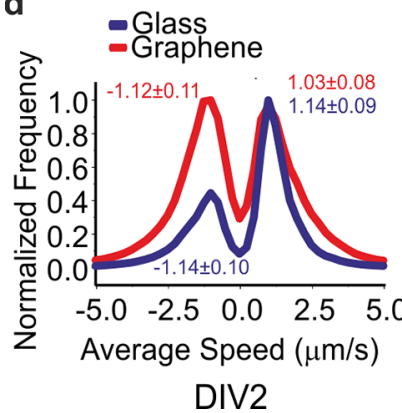

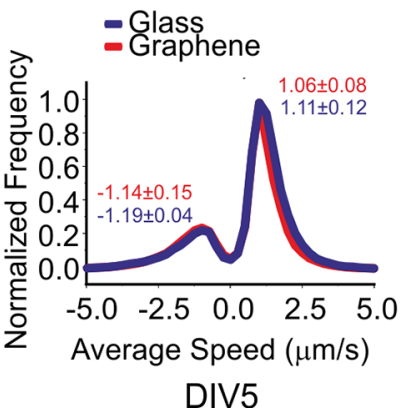

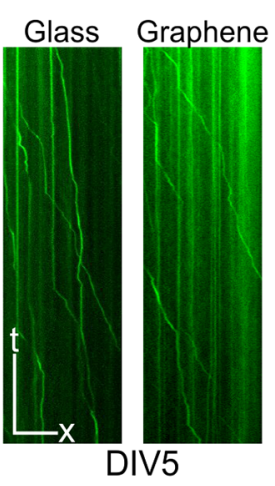

e
C

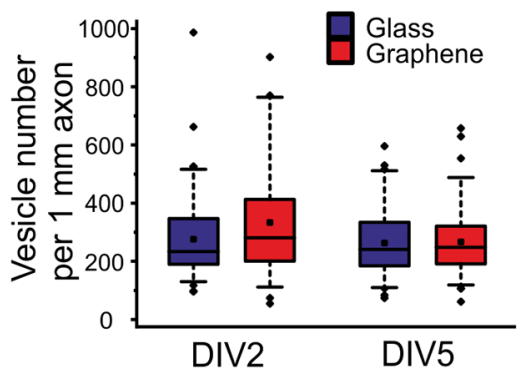

Figure 2. Altered axonal transport of fluoNGF in DRG neurons on graphene. (a) Schematic representation of compartmentalized microfluidic cell culture. Neurons are seeded in the soma compartment (SC) and extend their axon in the $150 \mu \mathrm{m}$ microchannels reaching the axonal side (AC), where fluoNGF is administered (green droplets). (b) Representative kymographs of fluoNGF vesicles along a single axon at DIV2 and DIV5; $x$ scale bar, $5 \mu \mathrm{m}$; $y$-scale bar, $10 \mathrm{~s}$. (c) Box plots for the number of vesicles per $1 \mathrm{~mm}$ of axons after the administration of fluoNGF at the axon compartment of DRG neurons cultured on graphene and glass at DIV 2 and DIV 5. Data are not significantly different according to a MannWhitney test. In box plots: Box between 25th and 75th percentile; horizontal line: median; whiskers: 5th and 95th percentiles; square: mean; circles: outliers. (d) Speed distributions for moving parts of vesicle trajectories on graphene (red) and glass (blue) at DIV2 and DIV5. The mean \pm standard deviation (SD) of each peak position calculated from the three independent replica is reported in the graph in the same color. Distributions with maxima normalized at 1 . (e) Percentage of vesicles in different categories at DIV2 and DIV5 (mean \pm s.e.m. for independent cultures). The four categories are stalled (stl), retrograde (ret), anterograde (ant), and oscillating (osc). The percentage of stalled and retrograde vesicles on graphene at DIV2 was significantly different from the control $(* * * p<0.0001$, one-way ANOVA, with Bonferroni's multiple comparison test). The distribution of the populations at DIV5 did not significantly differ, according to one-way ANOVA. The number of acquired time-lapse images used to calculate the vesicle population was 81 for glass and 56 for graphene at DIV2 and 75 for glass and 72 for graphene at DIV5. For all panels, the number of vesicles at DIV2 in three independent cultures was 3604 for graphene and 5188 for glass. The number of vesicles at DIV5 in two independent cultures was 4905 for graphene and 5399 for glass.

Furthermore, this analysis revealed an organized pattern of DRGs grown on graphene already at DIV3, whereby somas are clumped together and dense axonal bundles depart radially from them (Figure S1c,d). Knowing the influence of the substrate nanotopography on cell spreading, ${ }^{23,24}$ we studied graphene surface morphology via AFM (Figure S2). The flatness of the surface, with a root-mean-square roughness of about $1.8 \mathrm{~nm}$, made us exclude the presence of features that can lead to discontinuous cell-substrate adhesion. ${ }^{23}$ Moreover, as graphene crumpling was never observed in our analyses, we can exclude its effect on substrate wettability and soma clumping. ${ }^{23,25}$

Obtained results support the trend previously reported for embryonic DRG neurons on epitaxial graphene. ${ }^{4}$ Furthermore, the time-dependent axonal increase agrees well with what reported for non-compartmentalized hippocampal neurons on CVD graphene, where an initial $\sim 30 \%$ increased elongation was reported for DIV2, followed by a stabilization at $\sim 13 \%$ in the following days. ${ }^{1}$ It is thus established that graphene strongly impacts the early development of axon elongation in peripheral sensory neurons. The optical transparency of graphene makes it an ideal substrate to probe possible changes in uptake and trafficking of trophic factors, which are at the basis of axon elongation and neural survival.

Graphene Alters Retrograde Transport of Nerve Growth Factor Signaling Endosomes. Survival of sympathetic and sensory neurons is known to rely on the retrograde axonal transport of NGF signaling endosomes back to the cell body, ${ }^{17,18,26}$ while numerous studies indicate that axon elongation is prompted by a local effect of NGF which does not directly involve mechanisms in the cell body. ${ }^{27}$ Hence, we examined whether the observed graphene-induced axon outgrowth correlates with an altered NGF axonal transport. We used fluorescence microscopy to perform axonal transport studies in living, compartmentalized DRG neurons using a fluorolabeled NGF variant (fluoNGF) covalently coupled to an Alexa488 organic dye. ${ }^{28,29}$ A schematic of the experiment is shown in Figure $2 \mathrm{a}$.

To assess the effect of graphene on the transported vesicles, NGF vesicular trafficking was examined within the microchannels, using compartmentalized neurons on glass as control. Visual inspection of the detected vesicles suggested that graphene induces a strong reduction of retrogradely transported NGF vesicles at DIV2 (Supplementary Videos S1 and $\mathrm{S} 2$ ), while this is not the case at DIV5 (Figure 2b). To clarify if 


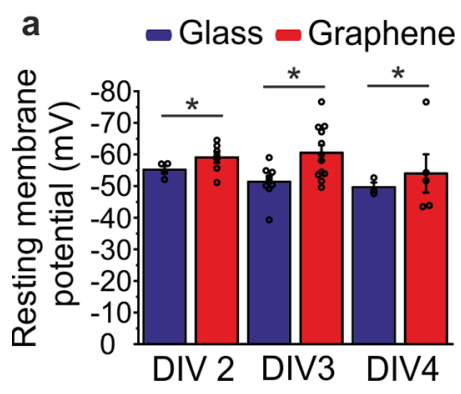

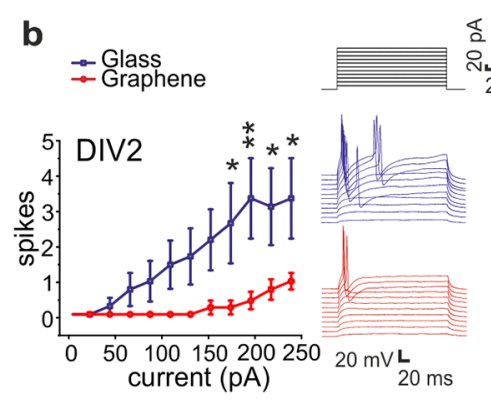

f

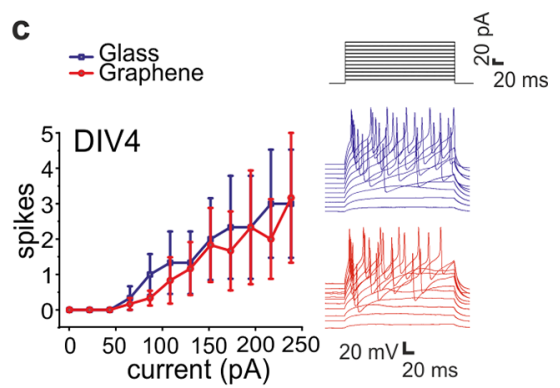

g

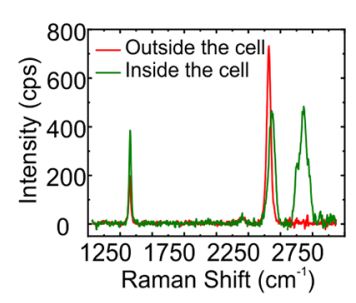

e

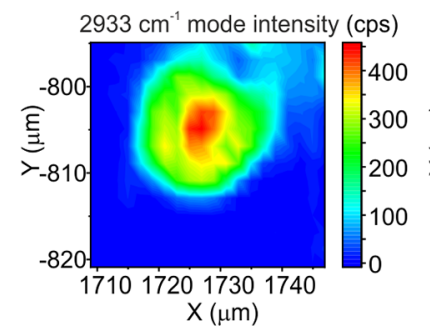

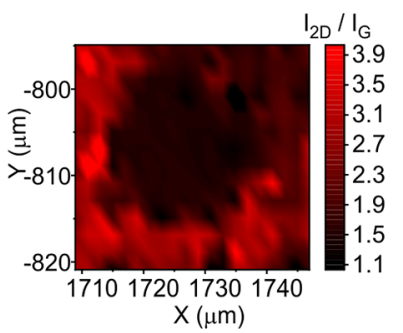

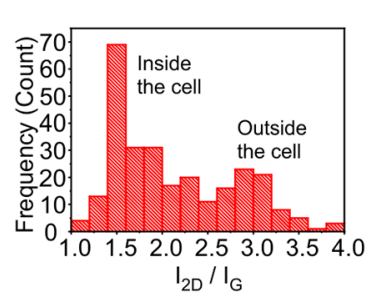

Figure 3. Altered neuron excitability and graphene electronic properties. (a) Graphene-cultured DRGs show hyperpolarized resting membrane potential in comparison to glass-cultured controls $\left({ }^{*} P_{\text {substrate }}<0.05\right.$, two-way ANOVA). The number of measured neurons, collected in three independent cultures, was: DIV2, glass $n=4$, graphene $n=7$; DIV3, glass $n=9$, graphene $n=12$; DIV4, glass $n=3$, graphene $n=5$. (b,c) Number of spikes in response to current injection and representative traces on graphene and glass-cultured DRG at different DIVs; voltage traces at the different injected currents shown in the top graphs are shifted vertically. (b) On DIV2, graphene-cultured DRGs show reduced spike number in response to current injection with respect to glass-cultured controls $\left(P_{\text {substrate }} \times\right.$ current $<0.001$, two-way ANOVA, with Holm-Sidak posthoc test, $* p$ $<0.05, * * p=0.005$ ). (c) On DIV4, no difference in spiking was observed between graphene- and glass-cultured DRGs according to two-way ANOVA test at 0.05 significance. The number of measured neurons, collected in three independent cultures, was: DIV2, glass $n=5$, graphene $n=$ 6; DIV4, glass $n=3$, graphene $n=6$. (d) Representative Raman spectra obtained on bare graphene (red line) and on the neuron-graphene interface at DIV2 (green line). (e) Raman map of the $2933 \mathrm{~cm}^{-1}$ mode intensity, identifying the cell position. (f) $2 \mathrm{D} / \mathrm{G}$ intensity ratio map of the same area reported in (e), revealing a shift of the ratio in the area underneath the cell. (g) Histogram of 2D/G intensity ratio showing the bimodal distribution of the intensity outside the neuron and the intensity underneath the neuron. The histogram reported in ( $\mathrm{g}$ ) shows a bimodal distribution, where the distribution of $2 \mathrm{D} / \mathrm{G}$ intensity ratio of bare graphene is peaked at 2.8 , while the ratio in case of the graphene underneath the neuron decreases down to 1.5 .

such a change is due to an altered NGF uptake on graphene, we quantified the number of vesicles per $\mathrm{mm}$ of axons after NGF administration (Figure 2c) in control and graphene and found no significant difference between the two groups, both at DIV2 and DIV5. This is in agreement with the reported unmodified neuron survival rate on graphene compared to the control. ${ }^{4}$ We next ruled out that the observed effect was due to a slowing down of vesicles and accordingly quantified the velocities during retrograde and anterograde motion of the vesicles on graphene and glass (represented by positive and negative velocities respectively, Figure $2 \mathrm{~d}$ ). We found that velocities of anterograde and retrograde transport were not altered by graphene both at DIV2 and DIV5. Also, we observed, similarly to what reported in refs 28 and 30, that on glass, vesicles underwent preferentially a retrograde movement, resulting in an asymmetric distribution. Surprisingly, on graphene, we observed a symmetric distribution at DIV2, indicating comparable retrograde and anterograde movements for NGF vesicles. On the contrary, at DIV5 vesicles on graphene and glass exhibited similar distributions of speeds with predominant retrograde transport. The obtained data prompted us to analyze the entire population of NGF vesicles and classify them based on their movement. We distinguished four different categories: (1) stalled (stl) when the vesicles did not move; (2) retrograde (ret) when the vesicles moved from the axon tip to the cell soma; (3) anterograde (ant) when the vesicles moved from the cell soma to the axon tip; and (4) oscillating (osc) when the movement of the vesicle switched between retrograde and anterograde within a limited space. We found that in graphene and control, the majority of NGF vesicles were stalled both at DIV2 and DIV5 (Figure 2e). However, on graphene at DIV2, the number of retrogradely transported vesicles was 8.5 -fold reduced with respect to the control, in favor of a $13 \%$ increase of the stalled vesicles (Table S3). When comparing the oscillating and anterograde populations, we found no significant difference between graphene and control. Hence, in the first developmental stage, the graphene effect on NGF transport is to reduce retrogradely moving vesicles in favor of a locally stalled population. Importantly, this effect almost disappears in more mature DRG cultures, where we found that the percentages of both stalled and moving vesicles were superimposable to the control (Figure 2e, right). This time-dependent fashion matches the predominant effect of graphene in axon elongation at early developmental phases (Figures 1 and S1), suggesting a key role for this trafficking alteration in the process. In order to elucidate the cause/effect links between graphene, NGF vesicle stall, and axon elongation, we next investigated possible electrophysiological and structural changes prompted by the material-neuron interface.

Altered Neuron Excitability and Electrostatic Interaction with Graphene. Electric activity has been recently associated with increased retrograde flux of signaling endosomes in hippocampal neurons ${ }^{31}$ and to inhibited axon outgrowth in adult sensory neurons. ${ }^{32}$ Hence, we carried out patch-clamp electrophysiology measurements ${ }^{33}$ to study the 


\section{Graphene}
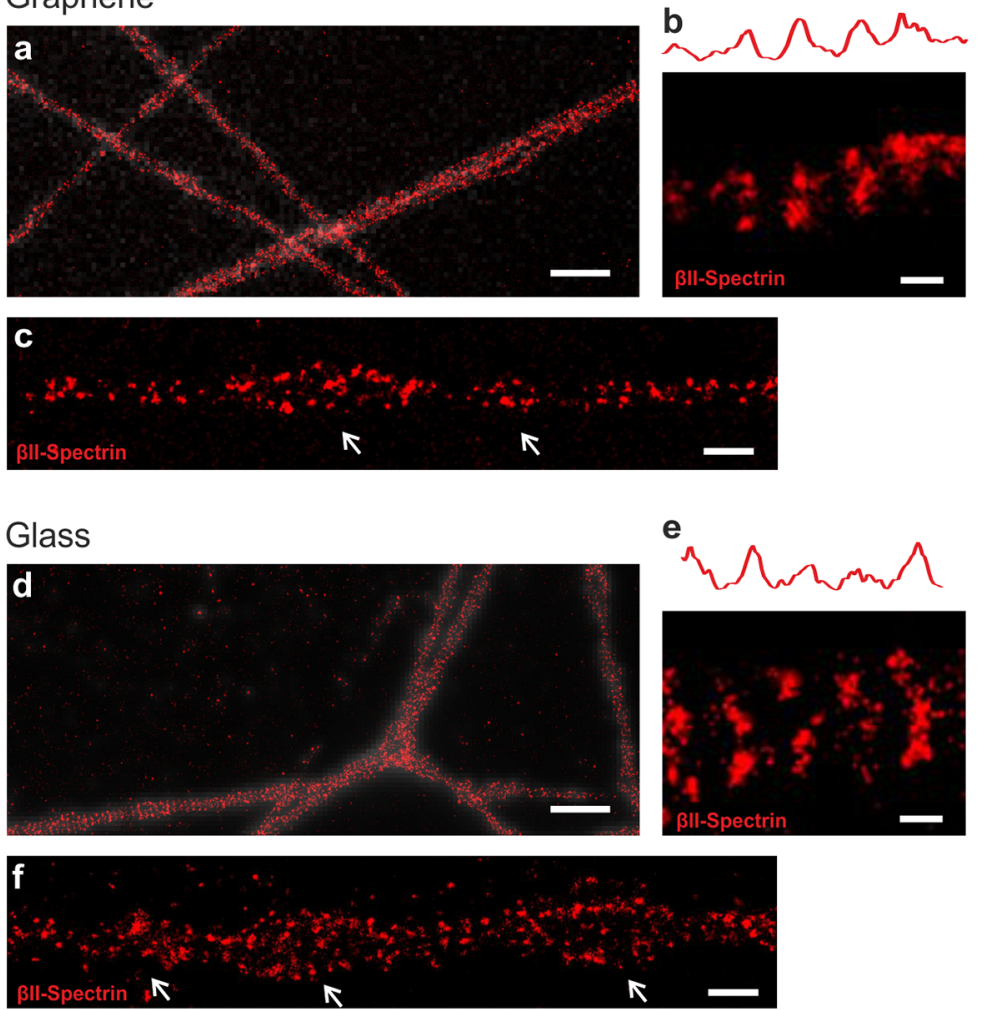

i

\section{Graphene}
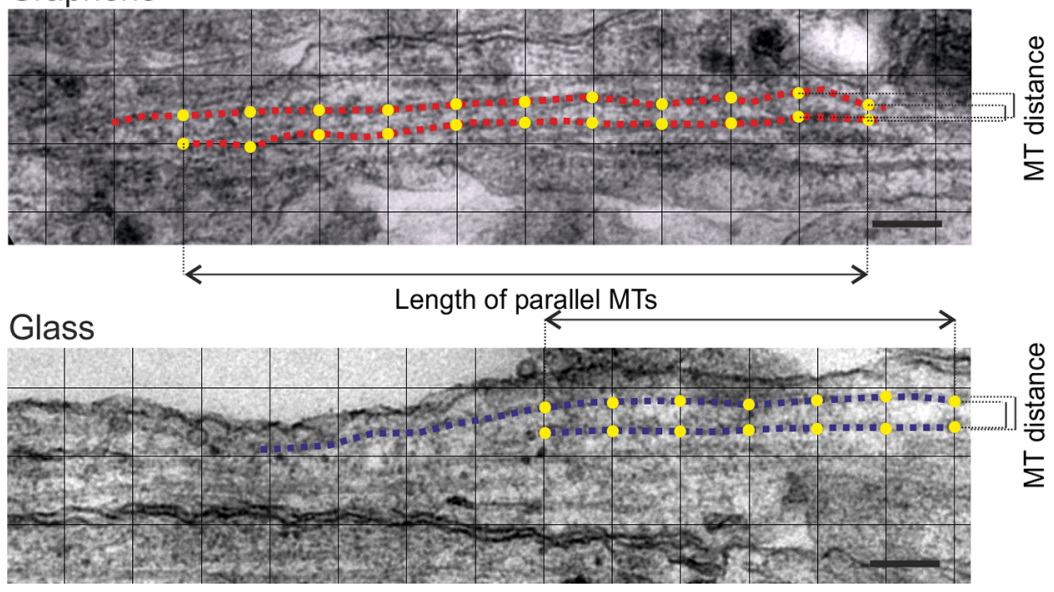

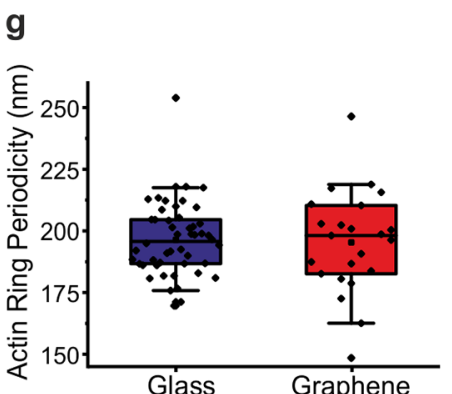

h
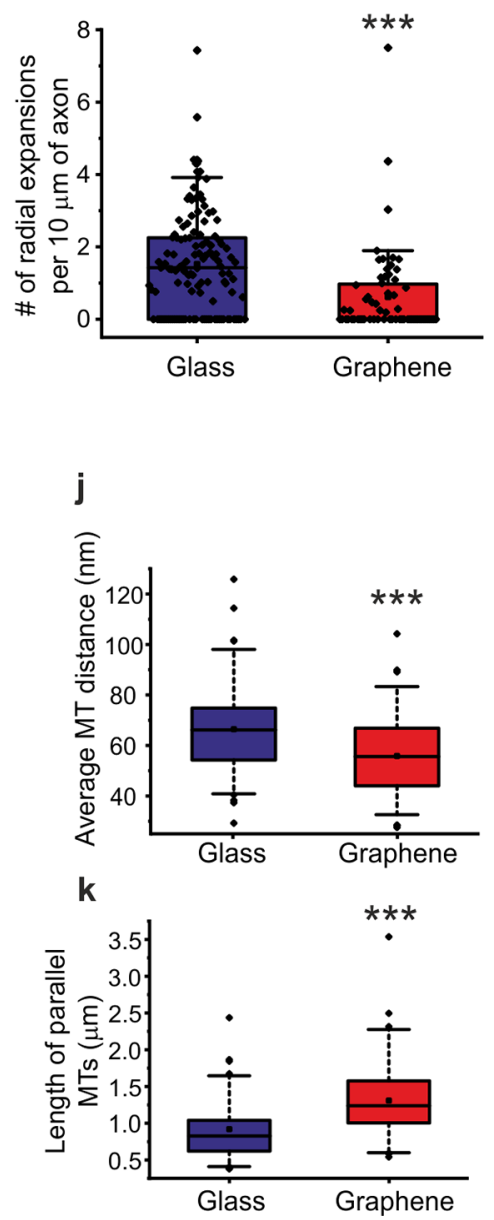

Figure 4. Modifications of axonal topology and cytoskeleton of DRGs on graphene. (a,d) Representative STORM images of $\beta$ II spectrin (red) in DRG neuron cultured on glass and on graphene and fixed at DIV2, superimposed to conventional fluorescence images (gray). Scale bar: $20 \mu \mathrm{m}$. $(\mathrm{b}, \mathrm{e})$ Magnification of the STORM images with intensity profiles across the periodic spectrin structure reported with a red line. Scale bar: $200 \mathrm{~nm}$. $(\mathrm{c}, \mathrm{f})$ Magnification of the STORM images with white arrows indicating MPS radial expansions (Scale bar: $500 \mathrm{~nm}$ ). (g) Box plots showing the periodicity of spectrin ring-like structure, which did not significantly differ according to Mann-Whitney Test. The number of analyzed regions is 21 for graphene and 48 for glass, each from two independent cultures. (h) Box plots of the number of radial expansions per $10 \mu \mathrm{m}$ of axon, showing a significantly higher number of radial expansions on glass than on graphene ( $* * *<0.0001$, Mann-Whitney Test). The number of analyzed axons is 64 for graphene and 123 for glass, each from two independent cultures. For the box plots in (g,h): Box between 25th and 75th percentile; horizontal line: median; whiskers: 5th and 95th percentiles; square: mean; circles: measured values. (i) Representative TEM images for quantifying axon diameter and number, geometry, and spacing of MTs of DRG cultured on graphene and glass. Scale bar: $200 \mathrm{~nm}$. A couple of adjacent MTs are highlighted with dotted red lines (graphene) or with dotted blue lines (glass). Grid-pattern lines are superimposed in black, and the intersection points of the grid lines with the MT, used to quantify the MT distance, are highlighted in yellow. The length of parallel MTs and the MT distance are also reported. ( $\mathrm{j}$ ) Box plots showing the average distance between a couple of microtubules within axons on glass and graphene $(* * * p<0.0001$, Mann-Whitney Test). (k) Box plots showing the lengths of parallel sections of microtubule couples inside the axon on glass and graphene $(* * * p<$ 0.0001, Mann-Whitney Test). For the box plots in (j,k): Box between 25th and 75th percentile; horizontal line: median; whiskers: 5th and 95th percentiles; square: mean; circles: outliers; number of analyzed couples: 79 for graphene and 97 for glass, each from two independent cultures. 
effect of graphene on DRG membrane resting potential and neuron excitability. To allow a direct comparison with the axonal elongation and transport studies, experiments were performed at similar time points. We observed a significantly hyperpolarized resting membrane potential for graphenecultured DRG neurons with respect to glass-cultured controls regardless of the DIV investigated. Cellular excitability was further investigated by quantifying the number of spikes evoked by current injection steps. We observed that, at DIV2, the mean number of spikes in graphene was significantly lower than in the control (Figure 3b). However, this difference disappeared at DIV4, with control and graphene neurons firing a similar number of spikes (Figure $3 \mathrm{c}$ ).

These results demonstrate that the early days of contact with graphene are characterized by a change in neuron excitability, followed by a phase of adaptation. Hence, we describe a correlation between neuron excitability, axon elongation, and signaling endosomes transport. The synchrony between restored excitability and recovery of normal NGF retrograde transport dynamics with prolonged culturing is a strong clue of such correlation. The recovery of normal signaling endosome transport shall, in turn, contribute to maintain neuronal survival. $^{34}$

In order to understand the reasons behind the observed reduced cellular excitability in the cell/electrolyte/graphene system, we carried out further electrical and spectroscopic analysis. Electrical measurements with a van der Pauw geometry of the graphene sample (i.e., coated and after medium immersion, with no neurons on top) indicate that holes are the majority carriers (Table S4). Peripheral neurons are known to be negatively charged, possessing more negative resting membrane potential with respect to central neurons. ${ }^{35}$ We thus investigated by Raman spectroscopy whether the local charge in graphene could be affected by a significant electrical interaction with the cell. Figure $3 \mathrm{~d}-\mathrm{g}$ shows the results obtained by carrying out Raman mapping of graphene with cultured DRG neurons at DIV2. The Raman spectrum measured for the neuron/graphene system (green line) presents, in addition to the well-known G and 2D Raman modes, ${ }^{36}$ a complex band peaked at $2933 \mathrm{~cm}^{-1}$, with one shoulder at $2885 \mathrm{~cm}^{-1}$ (Figure 3d). These Raman modes can be attributed to the cell and in particular to the $\mathrm{CH}_{3}$ stretching (proteins) and to the $\mathrm{CH}_{2}$ antisymmetric stretching (lipids), respectively. ${ }^{37}$ Indeed, Figure $3 \mathrm{e}$ shows the Raman map of the $2933 \mathrm{~cm}^{-1}$ mode intensity, identifying the cell position. The same areas were mapped to extract the $2 \mathrm{D} / \mathrm{G}$ intensity ratio map (Figure 3f). The intensity ratio was found to be decreased precisely underneath the neuron (Figure $3 \mathrm{~g}$ ), demonstrating an increase of the local hole doping of graphene (from $2 \times 10^{12}$ $\mathrm{cm}^{-2}$ to $6 \times 10^{12} \mathrm{~cm}^{-2}$ ), clearly ascribable to the cell negative membrane potential. This value is in agreement with the one reported for a graphene-astrocyte system. ${ }^{38}$

It is known that carbon-based $\pi$-electron-rich surfaces are ideal adsorption sites for potassium $\left(\mathrm{K}^{+}\right)$ions. $^{39,40}$ In particular, it was shown that the electronic properties of graphene can tune the efficiency of graphene in trapping $\mathrm{K}^{+}$ ions and that such trapping ability is likely to be significant in p-doped graphene (i.e., our case). ${ }^{11}$ This will reduce the extracellular concentration of potassium $\left(\mathrm{K}^{+}\right)$ions, thus affecting transmembrane ionic currents. Thus, we speculate that a modified (i.e., reduced) extracellular $\mathrm{K}^{+}$concentration due to preferential adsorption of $\mathrm{K}^{+}$ions may increase $\mathrm{K}^{+}$ currents and dampen neuronal excitability of graphene- cultured DRGs. At this time, the cause/effect link between altered excitability and modified NGF transport remains elusive, and further studies shall be performed to elucidate it. Since NGF transport could be also influenced by the axonal structure on graphene, in the next section, we examine the ultrastructure of axons grown on graphene at early developmental stage.

Reduced Microtubule Distance and Elongated Axonal Topology on Graphene. NGF retrograde axonal transport is mediated by the dynein-microtubule (MT) transport system ${ }^{41}$ and can thus be influenced by structural changes in the axon. This is maintained by a complex system of proteins including MTs and actin filaments that ensures shape, capacity to direct movements, and extension of the growth cone. ${ }^{42}$

Here, we first took the membrane-associated periodic skeleton (MPS) in consideration. This is a periodic ring-like structure around the axon circumference formed by actin, spectrin, and associated proteins, found in a broad range of neuronal cell types including DRG neurons. ${ }^{43,44}$ We examined the MPS with stochastic optical reconstruction microscopy (STORM), for studying possible variations induced by graphene. DRG neurons were fixed at DIV2 and immunostained for $\beta$ II spectrin for STORM imaging (Figure 4).

We found that graphene-cultured neurons maintain the characteristic periodicity of $\sim 190 \mathrm{~nm}$ of the MPS (graphene, $195.6 \pm 20.8 \mathrm{~nm}$ vs glass, $196.7 \pm 14.9 \mathrm{~nm}$, Figure $4 \mathrm{~g}$ ). However, the super-resolution approach also allowed us to distinguish the presence of regions in which the MPS expands radially (white arrows in Figure $4 c, f$ ). These are compatible either with zones of minor compaction within axonal bundles ${ }^{45}$ or with increased diameter of single axons. The number of MPS radial expansions was quantified over an axonal length of $10 \mu \mathrm{m}$, and we found that on graphene, they were more than halved with respect to the control (Figure $4 \mathrm{~h}$ ). These data confirm the observation that on graphene, axonal bundles are tightly organized already at the early developmental stage (Figure S1c,d), but also suggest that axons therein may have a stretched structure, which would well correlate with their increased length (Figure 1).

We next exploited transmission electron microscopy (TEM) to analyze whether and how the MT organization is affected by this topological rearrangement. In detail, we quantified the spacing between adjacent couples of MTs in longitudinal sections of the DRG culture on graphene in comparison with the control (Figure 4i), processing the neurons as described before. ${ }^{46}$ We found that on graphene the intratubular distance was significantly reduced by $19 \%$, when compared to the control (Figure $4 j$ ). In order to exclude an effect of the axon caliber on this measure, ${ }^{47}$ we quantified the mean axon diameter and the number of MTs per axon, which were found to be comparable in the two cases (Figure S5). Accordingly, a parallel immunofluorescence quantification of tubulin showed that the mean fluorescence intensity of Alexa488-labeled $\beta$ IIItubulin is similar for DRG cultures grown on graphene and control (Figure S6). Moreover, we measured the length for which the pairs of MTs arranged into adjacent arrays remains parallel along the same axon, finding that this parameter exhibits a remarkable $42 \%$ increase in graphene with respect to the control (Figure 4k), indicating that MTs are closer and straighter in axons on graphene than on the control substrate. This can be easily explained by the electrostatic interaction experienced by axons on graphene (Figure 3), which may 
increase their adhesion to the substrate, finally changing their three-dimensional architecture and forcing them to remain straighter than on the control substrate. Overall, our results suggest that the interaction between graphene and neurons does not dramatically alter the axonal ultrastructural organization, maintaining a similar total number and integrity of MTs and MPS, but rather confers to axons an elongated morphology. This effect may also provide another cause for the observed stall of NGF signaling endosomes (Figure 2). Indeed, transient radial expansions of axonal diameter were recently described to facilitate the processivity of the fast-moving retrograde carriers. ${ }^{48}$ Accordingly, the inhibition of these expansions prompted by graphene (Figure $4 \mathrm{~h}$ ), along with the reduced MT interdistance (Figure $4 \mathrm{j}$ ), might create a more crowded space that inhibits cargo mobility and reduces the retrograde transport of NGF-loaded endosomes, ultimately favoring their local action on axon outgrowth.

\section{CONCLUSIONS}

Graphene has attracted the interest of the neuroscience community worldwide for its biocompatibility, electrical properties, and regenerative potential. ${ }^{49-52}$ However, the real possibility of using this material for neuroregeneration applications will depend on our understanding of how graphene influences the processes of neurite outgrowth, elongation, and regeneration following injury. This can be achieved by exploiting graphene optical transparency to observe the processes of interest, as we propose in this work. Optical microscopy of DRG neurons shows an axonal length greater on graphene than in the control. Single-molecule fluorescence microscopy in living DRG neurons reveals that, although on graphene neurons are completely viable for weeks, ${ }^{4,11}$ deep nanoscale changes characterize the early cellmaterial interaction. In detail, we observe an 8.5-fold reduction of retrogradely transported vesicles of NGF, the signaling molecule known to mediate sensory axon development (Figure $2 \mathrm{e})$. These data are in line with previous observations that local NGF actions account for axon elongation. ${ }^{27}$ Indeed, NGFinduced axonal sprouting in sensory neurons has been widely investigated, and NGF treatment probed in models of spinal cord injury. ${ }^{53}$ Several aspects still need to be addressed, for example, whether graphene locally stalls only NGF vesicles, or also other fast-retrograde carriers, if NGF internalized at the soma is also influenced by graphene, and how the spatiotemporal signaling events triggered by NGF receptors ${ }^{54}$ change on graphene. Nevertheless, our experiments provide a clear clue that the physical control of the intracellular NGF pool may be useful for regeneration approaches.

To investigate possible causes for the observed NGF trafficking alteration, we studied graphene influence on both electrical and structural properties of axons. In the former case, we observe mutual effects: on one side, neurons change graphene charge concentration, inducing a doping clearly measurable via Raman spectroscopy (Figure 3f), and on the other side, graphene hyperpolarizes the resting membrane potential and reduces cell excitability (Figure $3 a-c$ ). It is noteworthy that this condition correlates with a maximal effect of graphene on axonal length (up to $>70 \%$ increase with respect to a control substrate, Tables 1 and S1) and was previously linked to increased axon regeneration in sensory neurons. $^{55}$ On the other hand, graphene also alters axon morphology, inducing longer and straighter axonal bundles
(Figures 1, S1, and 4h) and closer and straighter MTs within each axon (Figure $4 \mathrm{j}$ ).

The sum of these effects is progressively diminished with time, allowing to reach already after 4-5 DIV an excitability and axonal transport comparable to the control (Figures 2e and $3 \mathrm{~b}$ ) and stabilizing to a $17 \%$ increased axonal outgrowth (Figure $1 \mathrm{~b}$ and Table 1 ). Thus, the molecular players stalling NGF signaling endosomes are selectively switched on upon the early contact with the material. More studies are needed to understand if the electrophysiological and structural graphene effects can impact independently the transport of NGF vesicles, or if they are rather synergistic. This will depend on the ability to experimentally dissect membrane hyperpolarization, axonal stretching, and retrograde transport inhibition, while not precluding the correct NGF uptake to preserve neuron survival. Obtained results may surely increase our mechanistic detail of axonal growth processes and help to design optimal biocompatible guides to enhance them.

\section{ASSOCIATED CONTENT}

Supporting Information

The Supporting Information is available free of charge at https://pubs.acs.org/doi/10.1021/acs.nanolett.0c00571.

Experimental methods; morphology and axonal elongation in DRG neurons grown on graphene; AFM characterization of graphene; supplementary results on retrograde transport of vesicles containing NGF; measurements of carrier concentration in graphene; Raman characterization of graphene; TEM analysis of microtubule organization; DRG tubulin quantification (PDF)

Video S1: Visual inspection of the experimental acquisitions of fluorescent NGF on glass (AVI)

Video S2: Visual inspection of the experimental acquisitions of fluorescent NGF on graphene (AVI)

\section{AUTHOR INFORMATION}

\section{Corresponding Authors}

Domenica Convertino - NEST, Scuola Normale Superiore, 56127 Pisa, Italy; Center for Nanotechnology Innovation @ NEST, Istituto Italiano di Tecnologia, 56127 Pisa, Italy; ○ orcid.org/0000-0002-6115-9790;

Email: domenica.convertino@iit.it

Laura Marchetti - Center for Nanotechnology Innovation@ NEST, Istituto Italiano di Tecnologia, 56127 Pisa, Italy; Department of Pharmacy, University of Pisa, 56127 Pisa, Italy; Email: laura.marchetti@unipi.it

Camilla Coletti - Center for Nanotechnology Innovation @ NEST, Istituto Italiano di Tecnologia, 56127 Pisa, Italy; ○ orcid.org/0000-0002-8134-7633; Email: camilla.coletti@ iit.it

\section{Authors}

Filippo Fabbri - Center for Nanotechnology Innovation @ NEST, Istituto Italiano di Tecnologia, 56127 Pisa, Italy; (1) orcid.org/0000-0003-1142-0441

Neeraj Mishra - Center for Nanotechnology Innovation @ NEST, Istituto Italiano di Tecnologia, 56127 Pisa, Italy

Marco Mainardi - BIO@SNS Laboratory, Scuola Normale Superiore, 56126 Pisa, Italy

Valentina Cappello - Center for Nanotechnology Innovation @ NEST, Istituto Italiano di Tecnologia, 56127 Pisa, Italy 
Giovanna Testa - BIO@SNS Laboratory, Scuola Normale Superiore, 56126 Pisa, Italy

Simona Capsoni - BIO@SNS Laboratory, Scuola Normale Superiore, 56126 Pisa, Italy; Section of Physiology, Department of Biomedical and Specialty Surgical Sciences, University of Ferrara, 44121 Ferrara, Italy

Lorenzo Albertazzi - Nanoscopy for Nanomedicine Group, Institute for Bioengineering of Catalonia (IBEC), The Barcelona Institute of Science and Technology (BIST), 08024 Barcelona, Spain; Department of Biomedical Engineering, Institute for Complex Molecular Systems (ICMS), Eindhoven University of Technology, 5612AZ Eindhoven, The Netherlands

Stefano Luin - NEST, Scuola Normale Superiore, 56127 Pisa, Italy; NEST Istituto Nanoscienze, CNR and Scuola Normale Superiore, 56126 Pisa, Italy

Complete contact information is available at: https://pubs.acs.org/10.1021/acs.nanolett.0c00571

\section{Author Contributions}

${ }^{\mathrm{II}}$ These authors contributed equally to this work. C.C. and L.M. coordinated the project. L.M., C.C., and D.C. designed the study. N.M., D.C., and C.C synthesized and characterized graphene substrates. D.C., L.M., G.T., and S.C. established DRG cultures on graphene. D.C., S.L., and L.M. performed axonal transport measures and data analysis. F.F., D.C., and C.C. performed Raman studies and data analysis. M.M., D.C., and L.M. performed electrophysiology studies and data analysis. V.C. and D.C. performed TEM experiments and data analysis. D.C., L.A., L.M., and S.L. performed STORM experiments and data analysis. D.C., L.M., and C.C., with contributions from all authors, wrote the manuscript.

\section{Notes}

The authors declare no competing financial interest.

\section{ACKNOWLEDGMENTS}

We thank G. Signore and A. Moscardini for assistance in the NGF purification, R. Amodeo and L. Ceccarelli for Sfp synthase enzyme production, and $M$. T. Ciotti for the DRG harvesting and dissociation protocol. We thank A. Cattaneo, F. Beltram, V. Raffa, and C. Leterrier for useful discussions.

\section{REFERENCES}

(1) Li, N.; et al. The promotion of neurite sprouting and outgrowth of mouse hippocampal cells in culture by graphene substrates. Biomaterials 2011, 32, 9374-9382.

(2) Hong, S. W. Enhanced neural cell adhesion and neurite outgrowth on graphene-based biomimetic substrates. BioMed Res. Int. 2014, 2014, 212149.

(3) Meng, S. Nerve cell differentiation using constant and programmed electrical stimulation through conductive non-functional graphene nanosheets film. Tissue Eng. Regener. Med. 2014, 11, 274283.

(4) Convertino, D.; Luin, S.; Marchetti, L.; Coletti, C. Peripheral neuron survival and outgrowth on graphene. Front. Neurosci. 2018, 12, $1-8$.

(5) Lee, J. S.; et al. Graphene substrate for inducing neurite outgrowth. Biochem. Biophys. Res. Commun. 2015, 460, 267-273.

(6) Wang, L.; et al. Silk-Graphene Hybrid Hydrogels with Multiple Cues to Induce Nerve Cell Behavior. ACS Biomater. Sci. Eng. 2019, 5 , $613-622$.

(7) Zhang, C.; et al. Graphene trapped silk scaffolds integrate high conductivity and stability. Carbon 2019, 148, 16-27.

(8) Fu, C.; et al. Effect of electrical stimulation combined with graphene-oxide-based membranes on neural stem cell proliferation and differentiation. Artif. Cells, Nanomed., Biotechnol. 2019, 47, 18671876.

(9) Faroni, A.; Mobasseri, S. A.; Kingham, P. J.; Reid, A. J. Peripheral nerve regeneration: Experimental strategies and future perspectives. Adv. Drug Delivery Rev. 2015, 82, 160-167.

(10) Bramini, M.; et al. Interfacing Graphene-Based Materials With Neural Cells. Front. Syst. Neurosci. 2018, 12, 1-22.

(11) Pampaloni, N. P.; et al. Single-layer graphene modulates neuronal communication and augments membrane ion currents. Nat. Nanotechnol. 2018, 13, 755-764.

(12) Uesaka, N.; Hirai, S.; Maruyama, T.; Ruthazer, E.; Nobuhiko, Y. Activity Dependence of Cortical Axon Branch Formation: A Morphological and Electrophysiological Study Using Organotypic Slice Cultures. J. Neurosci. 2005, 25, 1-9.

(13) Singh, B.; et al. Accelerated axon outgrowth, guidance, and target reinnervation across nerve transection gaps following a brief electrical stimulation paradigm: Laboratory investigation. J. Neurosurg. 2012, 116, 498-512.

(14) Kalil, K.; Dent, E. W. Branch management: Mechanisms of axon branching in the developing vertebrate CNS. Nat. Rev. Neurosci. 2014, 15, 7-18.

(15) Bourke, J. L.; Coleman, H. A.; Pham, V.; Forsythe, J. S.; Parkington, H. C. Neuronal Electrophysiological Function and Control of Neurite Outgrowth on Electrospun Polymer Nanofibers Are Cell Type Dependent. Tissue Eng., Part A 2014, 20, 1089-1095.

(16) Lee, J. Y.; Bashur, C. A.; Gomez, N.; Goldstein, A. S.; Schmidt, C. E. Enhanced polarization of embryonic hippocampal neurons on micron scale electrospun fibers. J. Biomed. Mater. Res., Part A 2009, 92, 1398-1406.

(17) Snider, W. D.; Silos-Santiago, I. Dorsal root ganglion neurons require functional neurotrophin receptors for survival during development. Philos. Trans. R. Soc., B 1996, 351, 395-403.

(18) Lentz, S. I.; Knudson, C. M.; Korsmeyer, S. J.; Snider, W. D. Neurotrophins support the development of diverse sensory axon morphologies. J. Neurosci. 1999, 19, 1038-1048.

(19) Testa, G.; et al. The $\operatorname{NGF}(\mathrm{R} 100 \mathrm{~W})$ mutation specifically impairs nociception without affecting cognitive performance in a mouse model of Hereditary Sensory and Autonomic Neuropathy type V. J. Neurosci. 2019, 39, 9702-9715.

(20) Miseikis, V.; et al. Rapid CVD growth of millimetre-sized single crystal graphene using a cold-wall reactor. 2D Mater. 2015, 2, 014006.

(21) Barde, Y. A. Trophic factors and neuronal survival. Neuron 1989, 2, 1525-1534.

(22) Chowdary, P. D.; Che, D. L.; Zhang, K.; Cui, B. Retrograde NGF axonal transport - Motor coordination in the unidirectional motility regime. Biophys. J. 2015, 108, 2691-2703.

(23) Wang, L.; et al. Engineering subcellular-patterned biointerfaces to regulate the surface wetting of multicellular spheroids. Nano Res. 2018, 11, 5704-5715.

(24) Cai, P.; Zhang, X.; Wang, M.; Wu, Y. L.; Chen, X. Combinatorial Nano-Bio Interfaces. ACS Nano 2018, 12, 5078-5084.

(25) Zang, J.; et al. Multifunctionality and control of the crumpling and unfolding of large-area graphene. Nat. Mater. 2013, 12, 321-325.

(26) Ye, H.; Kuruvilla, R.; Zweifel, L. S.; Ginty, D. D. Evidence in support of signaling endosome-based retrograde survival of sympathetic neurons. Neuron 2003, 39, 57-68.

(27) Campenot, R. B. NGF and the local control of nerve terminal growth. J. Neurobiol. 1994, 25, 599-611.

(28) De Nadai, T.; et al. Precursor and mature NGF live tracking: One versus many at a time in the axons. Sci. Rep. 2016, 6, 20272.

(29) Di Matteo, P.; Calvello, M.; Luin, S.; Marchetti, L.; Cattaneo, A. An Optimized Procedure for the Site-Directed Labeling of NGF and proNGF for Imaging Purposes. Front. Mol. Biosci. 2017, 4, 1-9.

(30) Cui, B.; et al. One at a time, live tracking of NGF axonal transport using quantum dots. Proc. Natl. Acad. Sci. U. S. A. 2007, 104, 13666.

(31) Wang, T. Flux of signalling endosomes undergoing axonal retrograde transport is encoded by presynaptic activity and TrkB. Nat. Commun. 2016, 7, 12976. 
(32) Enes, J.; et al. Electrical activity suppresses axon growth through Cav1.2 channels in adult primary sensory neurons. Curr. Biol. 2010, 20, 1154-1164.

(33) Siano, G.; et al. Tau Modulates VGluT1 Expression. J. Mol. Biol. 2019, 431, 873-884.

(34) Vaillant, A. R.; et al. 3-Kinase - Akt Pathway to Synergistically Regulate Neuronal Survival. J. Cell Biol. 1999, 146, 955-966.

(35) Stein, V.; Nicoll, R. A. GABA Generates Excitement. Neuron 2003, 37, 375-378.

(36) Ferrari, A. C.; Basko, D. M. Raman spectroscopy as a versatile tool for studying the properties of graphene. Nat. Nanotechnol. 2013, $8,235-246$

(37) Caponi, S.; et al. Raman micro-spectroscopy: A powerful tool for the monitoring of dynamic supramolecular changes in living cells. Biophys. Chem. 2013, 182, 58-63.

(38) Keisham, B.; Cole, A.; Nguyen, P.; Mehta, A.; Berry, V. Cancer Cell Hyperactivity and Membrane Dipolarity Monitoring via Raman Mapping of Interfaced Graphene: Toward Non-Invasive Cancer Diagnostics. ACS Appl. Mater. Interfaces 2016, 8, 32717-32722.

(39) Williams, C. D.; Dix, J.; Troisi, A.; Carbone, P. Effective Polarization in Pairwise Potentials at the Graphene-Electrolyte Interface. J. Phys. Chem. Lett. 2017, 8, 703-708.

(40) Pham, T. A.; et al. Salt Solutions in Carbon Nanotubes: The Role of Cation- $\pi$ Interactions. J. Phys. Chem. C 2016, 120, 73327338.

(41) Chowdary, P. D.; Che, D. L.; Cui, B. Neurotrophin Signaling via Long-Distance Axonal Transport. Annu. Rev. Phys. Chem. 2012, 63, 571-594.

(42) Kevenaar, J. T.; Hoogenraad, C. C. The axonal cytoskeleton: from organization to function. Front. Mol. Neurosci. 2015, 8, 1-12.

(43) Xu, K.; Zhong, G.; Zhuang, X. Actin, Spectrin, and Associated Proteins Form a Periodic Cytoskeletal Structure in Axons. Science (Washington, DC, U. S.) 2013, 339, 452-456.

(44) He, J.; et al. Prevalent presence of periodic actin-spectrinbased membrane skeleton in a broad range of neuronal cell types and animal species. Proc. Natl. Acad. Sci. U. S. A. 2016, 113, 6029-6034.

(45) Hauser, M.; et al. The Spectrin-Actin-Based Periodic Cytoskeleton as a Conserved Nanoscale Scaffold and Ruler of the Neural Stem Cell Lineage. Cell Rep. 2018, 24, 1512-1522.

(46) Linetti, A.; et al. Cholesterol reduction impairs exocytosis of synaptic vesicles. J. Cell Sci. 2010, 123, 595-605.

(47) Fournier, A. J.; et al. Changes in neurofilament and microtubule distribution following focal axon compression. PLoS One 2015, 10, e0131617.

(48) Wang, T.; et al. Radial contractility of Actomyosin-II rings facilitates cargo trafficking and maintains axonal structural stability following cargo-induced transient axonal expansion. Biofuels, Bioprod. Biorefin. 2019, 13, 1085.

(49) Fraczek-Szczypta, A. Carbon nanomaterials for nerve tissue stimulation and regeneration. Mater. Sci. Eng., C 2014, 34, 35-49.

(50) Jakus, A. E.; et al. Three-dimensional printing of high-content graphene scaffolds for electronic and biomedical applications. ACS Nano 2015, 9, 4636-4648.

(51) Liu, X.; et al. Functionalized Carbon Nanotube and Graphene Oxide Embedded Electrically Conductive Hydrogel Synergistically Stimulates Nerve Cell Differentiation. ACS Appl. Mater. Interfaces 2017, 9, 14677-14690.

(52) Qian, Y. An integrated multi-layer 3D-fabrication of PDA/RGD coated graphene loaded PCL nanoscaffold for peripheral nerve restoration. Nat. Commun. 2018, 9, 323.

(53) Keefe, K. M.; Sheikh, I. S.; Smith, G. M. Targeting neurotrophins to specific populations of neurons: NGF, BDNF, and NT-3 and their relevance for treatment of spinal cord injury. Int. J. Mol. Sci. 2017, 18, 548.

(54) Marchetti, L.; et al. Ligand-induced dynamics of neurotrophin receptors investigated by single-molecule imaging approaches. Int. J. Mol. Sci. 2015, 16, 1949-1979.

(55) Tedeschi, A.; He, Z. Axon regeneration: Electrical silencing is a condition for regrowth. Curr. Biol. 2010, 20, R713-R714.

\section{NOTE ADDED AFTER ASAP PUBLICATION}

This paper published ASAP on April 13, 2020 with errors in Figures 2, 3, and 4. The figures were replaced and the revised paper was posted on April 14, 2020. 